3 Research S Quare

\title{
Dexmedetomidine suppresses sevoflurane anesthesia-induced neuroinflammation through activating PI3K/Akt/mTOR pathway
}

\section{Nan Wang}

Department of Anesthesiology, Cancer Hospital of China Medical University, Liaoning Cancer Hospital \& Institute, Dalian Medical University Clinical Oncology College

Mingyu Wang ( $\triangle$ mingyuwang1211@163.com )

Department of Anesthesiology, Cancer Hospital of China Medical University, Liaoning Cancer Hospital \& Institute, Dalian Medical University Clinical Oncology College https://orcid.org/0000-0002-6557-2726

\section{Research Article}

Keywords: Dexmedetomidine, sevoflurane, neuroinflammation, PI3K/Akt/mTOR pathway

Posted Date: January 9th, 2019

DOl: https://doi.org/10.21203/rs.2.194/v1

License: (c) (i) This work is licensed under a Creative Commons Attribution 4.0 International License. Read Full License 


\section{Abstract}

Background: Sevoflurane, an inhalational general anesthetic, has become one of the most widely used inhalational anesthetics in surgery. However, previous studies have found that sevoflurane anesthesia can trigger an inflammatory response, resulting in secondary damage. Dexmedetomidine (DEX), a highlyselective $a$ adrenergic receptor agonist, is widely used as an anesthesia adjuvant drug in the clinic. Here, we investigate whether DEX can suppress sevoflurane anesthesia-induced neuroinflammation.

Methods: We sought to determine the mechanism of action of this suppressive effect using rats as a model. Rats were randomly divided into control group $(n=10)$, low-dose sevoflurane group ( $L-S e v ; n=10)$, high-dose sevoflurane group (H-Sev; $n=10)$, vehicle group $(n=10)$ and DEX group $(n=10)$. Western blot was used to detect the expression of proinflammatory cytokines (IL-6, IL-8, TNF-a) and the activity level of the phosphatidylinositol 3-hydroxy kinase/protein kinase B/mammalian target of rapamycin (PI3K/Akt/mTOR) pathway.

Results: We find that sevoflurane anesthesia induces an increase in the levels of pro-inflammatory cytokines, while decreasing activation of the PI3K/Akt/mTOR pathway in both the cortex and hippocampus of rats. Moreover, treatment with DEX reduced pro-inflammatory cytokine levels and prevented inactivation of the PI3K/Akt/mTOR pathway.

Conclusions: These data suggest that the PI3K/Akt/mTOR pathway could contribute to sevofluraneinduced neuroinflammation, and that activation of PI3K/Akt/mTOR signaling by DEX could help to reduce the neuroinflammation effects of sevoflurane.

Keywords: Dexmedetomidine, sevoflurane, neuroinflammation, PI3K/Akt/mTOR pathway

\section{Background}

In recent years the inhalational anesthetic sevoflurane has replaced other volatile anesthetics due to its mild irritation, quick induction, stable maintenance and rapid revival ${ }^{[1,2]}$. However, recent evidence has shown that sevoflurane can lead to pathophysiological alterations of brain in the recovery period, such as neuronal apoptosis, tau aggresomes, abnormal discharge of neurons, and neuroinflammation, which cause neurodegenerative changes in the development of mammalian brain ${ }^{[3-7]}$. Exposure to anesthesia in childhood may lead to adverse neurodevelopmental outcomes in children ${ }^{[8]}$. Despite evidence linking inhalational anesthesia to neurodegenerative effects, it is still considered safer than other anesthesia methods, and more than 3 million children undergo inhaled anesthesia every year ${ }^{[9,10]}$. Therefore, it is urgent to search for anesthesia adjuvants to reduce the neurotoxicity of inhaled anesthetics.

Dexmedetomidine (DEX), a selective a2-adrenoceptor agonist, is used clinically as an adjuvant of local anesthetics used in peripheral nerve blocks, where it prolongs the duration of sensory and motor blockades without causing toxicity ${ }^{[11,12]}$. In addition, DEX is able to cross the blood brain barrier and stimulate a2-adrenoceptors centrally and has been shown to exert neuroprotective effect ${ }^{[13]}$. Wang et al. 
found that DEX treatment could inhibit the expression of inflammatory cytokines and their mediators, resulting in reduced focal cerebral ischemia-reperfusion injury in rats. This data suggested that inhibition of the nuclear factor-kB pathway (NF-KB) may be a mechanism underlying the neuroprotective action of DEX ${ }^{[14]}$. Treatment with DEX after subarachnoid haemorrhage (SAH) attenuated SAH-induced early brain injury, partially through suppression of the toll like receptor 4 (TLR4)/NF-KB pathway and the NLRP3 inflammasome ${ }^{[15]}$. Additionally, DEX has been shown to improve post-operative cognitive dysfunction in aged mice by inhibition of the hippocampal inflammatory response and reduction of neuronal apoptosis [16]. Taken together, these data suggest that DEX has anti-inflammatory properties in the central nervous system (CNS).

Given the inflammation induced by sevoflurane and the anti-inflammatory properties of DEX, previous research has been conducted to determine whether DEX could provide protection from sevoflurane anesthesia-induced inflammation. In the present study, we investigated the molecular mechanism responsible for the protective effect of DEX against sevoflurane-induced inflammation in the CNS. We find that this inflammation is closely associated with the activity of the phosphatidylinostitol 3-hydroxy kinase/ protein kinase B/ mammalian target of rapamycin (PI3K/Akt/mTOR) pathway, and that treatment with DEX can prevent disruption of PI3K/Akt/mTOR pathway signaling.

\section{Methods}

\section{Animals}

Fifty healthy Sprague-Dawley (SD) rats, weighing 150-200 g were purchased from the Animal Experiment Center of Institute of Radiation Medicine of the Chinese Academy of Medical Sciences, China. The rats were fed food and water under specific pathogen-free conditions and kept in a $12 \mathrm{~h}$ light/dark cycle at 22$24^{\circ} \mathrm{C}$. All animal procedures were approved by The Institute of Radiation Medicine of the Chinese Academy of Medical Sciences and conducted in accordance with the ethical principles for Experiments on Animals as well as international standards.

\section{Groups and treatments}

Rats were randomly divided into five groups: control group ( $n=10)$, low-dose sevoflurane group (L-Sev; $n=10)$, high-dose sevoflurane group (H-Sev; $n=10)$, vehicle group $(n=10)$ and DEX group $(n=10)$. All rats were placed in the anesthesia box and received anesthesia using an inhalation machine. Oxygen concentration and anesthesia doses were continuously monitored. The control group was treated with $60 \% \mathrm{O}_{2}$ for $2 \mathrm{~h}$, L-Sev group was treated with $1.5 \%$ sevoflurane inhalation for $2 \mathrm{~h}$, and the remaining groups (L-Sev group, vehicle group, and DEX group) were treated with $3 \%$ sevoflurane inhalation for $2 \mathrm{~h}$ [2]. One hour before sevoflurane treatment the DEX group received intraperitoneal injection of $4 \mu \mathrm{g} / \mathrm{kg}$ and the vehicle group received intraperitoneal injection of saline ${ }^{[17]}$. The anesthesia time commenced when the sevoflurane concentration reached the maximum for each group. Gas flow in the anesthesia chamber was maintained at a rate of $4 \mathrm{~L} / \mathrm{min}$. 


\section{Tissue preparation}

At the end if the experiment, rats were sacrificed under anesthesia using $50 \mathrm{mg} / \mathrm{kg}$ sodium pentobarbital by intraperitoneal injection. The brain tissue was removed and a subset of brain samples was used to make $10 \mu \mathrm{m}$ sections for use in immunofluorescence staining. The remaining brains were placed on tin foil and the cortical and the hippocampal tissues were separated and stored at $-80{ }^{\circ} \mathrm{C}$ for western blot.

\section{Immunofluorescence staining}

Frozen brain sections were treated with ice cold acetone for 15 minutes, followed by incubation in $1 \%$ Triton-X for 20 min at room temperature. After blocking with 10\% goat serum (Beyotime, China) for 30 min at $37^{\circ} \mathrm{C}$, sections were incubated with rabbit anti-PI3K (1:100, CST, USA), rabbit anti-Akt (1:100, CST, USA), rabbit anti-mTOR (1:50, CST, USA) and mouse anti-NeuN (1:200, Millipore, USA) overnight at $4{ }^{\circ} \mathrm{C}$. Sections were washed with PBS and incubated with goat anti-rabbit Alexa Fluor cy3 (1:50, Beyotime, China) and goat anti-mouse Alexa Fluor 488. After washing with PBS, cell nuclei were then stained with DAPI (1:100, Beyotime, China). Fluorescence images were captured via laser scanning confocal microscopy (Nikon).

\section{Western blot analysis}

Cortical and hippocampal tissue samples were lysed and total protein was extracted using whole-cell protein extraction kits (Beyotime, China) according to the manufacturer's protocols. A bicinchoninic acid (BCA) protein quantification kit (Beyotime, China) was used to measure the protein concentration of the samples. The proteins were separated with $10 \%$ SDS-PAGE and transferred to polyvinylidene difluoride (PVDF) membranes. PVDF membranes were blocked in 2\% BSA in TBST at room temperature for 2 hours. Membranes were then incubated with primary antibodies overnight at $4^{\circ} \mathrm{C}$. The primary antibodies used in our study include rabbit anti-IL-6 (1:1000, Abcam, USA), IL-8 (1:1000, Abcam, USA), TNFa (1:1000, Abcam, USA), PI3K (1:1000, CST, USA), p-PI3K (1:1000, CST, USA), Akt (1:1000, CST, USA), p-Akt (1:1000, CST, USA), mTOR (1:1000, CST, USA), p-mTOR (1:1000, CST, USA) and GAPDH (1:4000, Proteintech, USA). Following staining with primary antibody, three TBST washes were preformed and membranes were incubated with a horseradish peroxidase-conjugated goat anti-rabbit IgG (1:4000, Proteintech, USA) for $2 \mathrm{~h}$ at room temperature. PVDF membranes were then washed three times with TBST and developed using ECL Plus reagent.

\section{Statistical Analysis}

All values are presented as the mean \pm SEM. Comparisons between groups were made using t-tests and one-way ANOVA followed by a post hoc Tukey test. Differences were considered to be statistically significant at the level of $P<0.05$.

\section{Results}


To determine the effect of sevoflurane on neuroinflammation, expression levels of the inflammatory markers IL-6, IL-8 and TNF-a were measured in the cortex and hippocampus of rats in the control, L-Sev and $\mathrm{H}-\mathrm{Sev}$ groups by western blot. IL-6, IL-8 and TNF- $a$ expression was significantly increased in the cortex and hippocampus after low-dose or high-dose sevoflurane anesthesia compared with that of the control group (Figure1, $\mathrm{p}<0.05$ ). Moreover, the high-dose sevoflurane group showed significantly higher levels of IL-6, IL-8 and TNF- $a$ than the low-dose group, indicating that the induction of inflammation was dose dependent (Figure 1, $p<0.05$ ).

\section{Effect of sevoflurane on the PI3K/Akt/mTOR pathway}

We first used immunofluorescence to determine the expression pattern of PI3K, Akt, and mTOR in the brain. We found that PI3K/Akt/mTOR pathway components were widely expressed in both neurons and other non-neuronal cells (Figure 2), indicating that the PI3K/Akt/mTOR pathway might play an important role in the physiological control of numerous functions of the central nervous system. Following this initial characterization, we assayed the levels of PI3K, Akt, and mTOR expression, as well as their phosphorylation status, in the cortex and hippocampus of rats from the control, L-Sev, and H-Sev groups to assess the effect of sevoflurane anesthesia on PI3K/Akt/mTOR signaling. As shown in Figure 3, both low-dose and high-dose sevoflurane treatment groups showed significantly decreased levels of $\mathrm{PI3K} / A k t / m T O R$ pathway phosphorylation $(\mathrm{p}<0.05)$. Moreover, the level of PI3K/AKT/mTOR pathway activity was significantly lower in the cortex and hippocampus after high-dose sevoflurane anesthesia compared with that of L-Sev group (Figure 3, p<0.05).

\section{Effect of dexmedetomidine on sevoflurane anesthesia-induced neuroinflammation}

To determine if dexmedetomidine could reduce neuroinflammation induced by sevoflurane anesthesia we measured the levels of IL-6, IL-8 and TNF- $a$ in the cortex and hippocampus of rats in the vehicle and DEX groups. Rats treated with DEX exhibited decreased levels of IL-6, IL-8, and TNF- $a$ compared to the vehicle control group, suggesting that DEX can inhibit sevoflurane anesthesia-induced neuroinflammation (Figure $4, p<0.05)$.

\section{Effect of dexmedetomidine on the PI3K/Akt/mTOR pathway}

Using western blot, we assayed the effects of DEX on PI3K/Akt/mTOR pathway activation. As shown in figure 5, the levels of PI3K, Akt, and mTOR phosphorylation were significantly increased in the cortex and hippocampus in the DEX group compared with vehicle group, indicating DEX could activate the $\mathrm{PI3K} / \mathrm{Akt} / \mathrm{mTOR}$ pathway under sevoflurane anesthesia $(p<0.05)$.

\section{Discussion}

In the present study, we observed that sevoflurane anesthesia treatment induced an increase in the expression of proinflammatory factors and led to a decrease in PI3K/Akt/mTOR pathway activity in both the cortex and hippocampus of rats. Additionally, we found that DEX treatment could restore 
$\mathrm{PI3K} / \mathrm{Akt} / \mathrm{mTOR}$ activity in rats treated with sevoflurane anesthesia. Thus, we propose that DEX can suppress sevoflurane anesthesia-induced neuroinflammation by modulating PI3K/Akt/mTOR pathway activity.

Postoperative neuroinflammation is a common pathological phenomenon in the CNS, and can lead to secondary damage, such as delirium, cognitive dysfunction, Alzheimer's disease, and other detrimental effects ${ }^{[18,19]}$. A healthy immune response is crucial for proper wound healing and repair of tissue damage, as well as to combat infection without harming the host's own cells or tissue. In contrast, excessive inflammatory responses are harmful, and can result in severe tissue damage, and even death $[18,20]$. Inhalational anesthetics, such as isoflurane and sevoflurane, can trigger pathological immune responses during surgery. Isoflurane, which has been used since the 1980s, is metabolized slowly leading to reduced induction of anesthesia during surgery and shorter recovery times ${ }^{[21]}$. Sevoflurane began to be used a decade later and has a lower blood-gas partition coefficient than the other anesthetics, leading to rapid induction of anesthesia and faster recovery times after anesthesia ${ }^{[22,23]}$. Several decades of research have been conducted on the toxicity and side effects of these volatile anesthetics. Here, we investigated the relationship between sevoflurane anesthesia and neuroinflammation. We used low- or high-does sevoflurane to treat rats, and then detected the expression of proinflammatory cytokines (IL-6, IL-8, TNF-a). We confirmed that sevoflurane could increase the levels of IL-6, IL-8 and TNF-a in the cortex and hippocampus of anesthetized rats, in agreement with previous research which indicated that either isoflurane or sevoflurane can increase IL-6 levels via activation of NF-KB signalling [24].

The PI3K/Akt/mTOR cascade is important in mediating the release of proinflammatory cytokines. PI3K is a ubiquitous lipid kinase which plays a crucial role in signal transduction through receptor tyrosine kinases. PI3K phosphorylates phosphatidylinositol-4,5-bis-phosphate (PIP2) to form phosphatidylinositol4,5-tri-phosphate (PIP3); PIP3 recruits other downstream molecules, such as serine-threonine kinases, including the major effector of PI3K activation, Akt ${ }^{[25]}$. Activated Akt can then in turn activate mTOR, leading to the phosphorylation of two downstream effectors, p70 ribosomal protein S6 kinase 1 (S6K1)

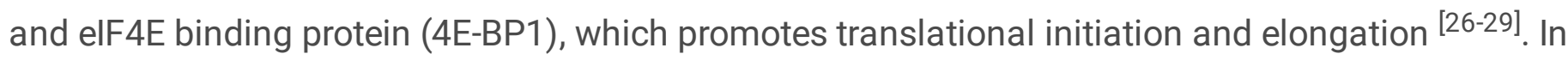
addition to its role in protein synthesis, mTOR also regulates the ubiquitin-proteasome system (UPS) ${ }^{[29,}$ 30]. The UPS is an important regulator of NF-KB signaling, as the inhibitory regulator of NF-KB must be degraded by the proteasome in order for NF-kB to become active. Rebecca et al. suggested that inhibition of mTOR by rapamycin could increase polyubiquitination and ubiquitin-mediated degradation of IKB-a, leading to NF-KB-induced transcriptional activation. This result suggested that the activation of $m T O R$ could prevent the occurrence of inflammation through suppression of NF-KB activation ${ }^{[31]}$, as NF-KB activity promotes the expression of proinflammatory cytokines ${ }^{[32,33]}$. We observed an increase in proinflammatory cytokine release after treatment with sevoflurane anesthesia, and this release of cytokines was accompanied by the inactivation of the PI3K/Akt/mTOR pathway. In addition, we observed widespread expression of PI3K, Akt, and mTOR in neurons and non-neuronal cells, which indicated that $\mathrm{PI3K} / \mathrm{Akt} / \mathrm{mTOR}$ pathway might be involved in the physiological control of numerous functions of the central nervous system. In sum, these data suggest that the inactivation of PI3K/Akt/mTOR pathway 
may be associated with sevoflurane anesthesia-induced neuroinflammation. Thus, increasing $\mathrm{PI} 3 \mathrm{~K} / \mathrm{Akt} / \mathrm{mTOR}$ pathway activity may be a promising and novel therapeutic strategy against postoperative neuroinflammation.

Recent studies have suggested that DEX has anti-inflammatory properties. Chen et al., for example, found that DEX treatment could suppress retinal ischemia/reperfusion injury, and showed effective antiinflammatory effects through inhibition of toll-like receptor 4 (TLR4)/NF-KB expression ${ }^{[34]}$. Additionally, Huang et al. also suggested that DEX could inhibit the nuclear translocation and binding activity of activated NF-KB, thus reducing inflammatory cytokines ${ }^{[34]}$. Given the anti-inflammatory properties of DEX and its effects on the PI3K/Akt/mTOR signaling pathway, we hypothesized that DEX could suppress sevoflurane anesthesia-induced neuroinflammation. Surprisingly, we found that treatment with DEX could down-regulate pro-inflammatory cytokines (IL-6, IL-8 and TNF-a) and up-regulate phosphorylated levels of $\mathrm{PI} 3 \mathrm{~K} / \mathrm{Akt} / \mathrm{mTOR}$, as a previous study indicated that DEX exerts an anti-inflammatory effect via the activation of $\mathrm{PI} 3 \mathrm{~K} / \mathrm{Akt} / \mathrm{mTOR}$ signaling in rats with traumatic brain injury ${ }^{[35]}$. Thus, we propose that DEX can ameliorate sevoflurane anesthesia-induced neuroinflammation through activation of the $\mathrm{PI3K} /$ Akt/mTOR signaling pathway.

\section{Conclusions}

Sevoflurane anesthesia induces neuroinflammation in the CNS, and this inflammation may be the result of decreased signaling through the PI3K/Akt/mTOR pathway. The administration of DEX can reduce neuroinflammation caused by sevoflurane, providing an important reference basis for clinical anesthesia.

\section{Abbreviations}

DEX Dexmedetomidine

PI3K/Akt/mTOR Phosphatidylinositol 3-hydroxy kinase/protein kinase B/mammalian target of rapamycin

NF-kB Nuclear factor-kB pathway

SAH Subarachnoid haemorrhage

TLR4 Toll like receptor 4

PIP2 Phosphatidylinositol-4,5-bis-phosphate

PIP3 Phosphatidylinositol-4,5-tri-phosphate

S6K1 S6 kinase 1

UPS Ubiquitin-proteasome system 


\section{Declarations}

\section{Ethics approval and consent to participate}

All animal procedures were approved by the Institute of Radiation Medicine of the Chinese Academy of Medical Sciences and conducted in accordance with the ethical principles for Experiments on Animals as well as international standards.

\section{Consent for publication}

Not applicable

\section{Availability of data and materials}

The datasets used and/or analyzed during the current study are available from the corresponding author on a reasonable request.

\section{Competing interests}

The authors declare that they have no competing interests.

\section{Funding}

No funding was received.

\section{Authors' contributions}

Research idea and project design: MW. Data collection and dataset setup: NW. Data analysis: NW. All authors contributed to the development of the manuscript, and approved the final version.

\section{Acknowledgments}

We thank members of our laboratory for technical help.

\section{References}

1 Tang L, Liu H, Wu Y, Li M, Li W, Jiang M, et al. Sevoflurane may be more beneficial than propofol in patients receiving endoscopic variceal ligation and endoscopic variceal sclerotherapy: A randomized, double-blind study. Exp Ther Med. 2017; 14:3145-52.

2 Cui RS, Wang K, Wang ZL. Sevoflurane anesthesia alters cognitive function by activating inflammation and cell death in rats. Exp Ther Med. 2018ه15:4127-30.

3 Huang L, Huang K, Ning H. Autophagy induction by hispidulin provides protection against sevofluraneinduced neuronal apoptosis in aged rats. Biomed Pharmacother. 2018ه98:460-8. 
4 Wang L, Zheng M, Wu S, Niu Z. MicroRNA-188-3p is involved in sevoflurane anesthesia-induced neuroapoptosis by targeting MDM2. Mol Med Rep. 2018囚17:4229-36.

5 Tao G, Zhang J, Zhang L, Dong Y, Yu B, Crosby G, et al. Sevoflurane induces tau phosphorylation and glycogen synthase kinase $3 \beta$ activation in young mice. Anesthesiology. 2014囚121:510-27.

6 Liang P, Li F, Liu J, Liao D, Huang H, Zhou C. Sevoflurane activates hippocampal CA3 kainate receptors (Gluk2) to induce hyperactivity during induction and recovery in a mouse model. Br J Anaesth. 2017区 119:1047-54.

7 Li D, Liu L, Li L, Li X, Huang B, Zhou C, et al. Sevoflurane Induces Exaggerated and Persistent Cognitive Decline in a Type II Diabetic Rat Model by Aggregating Hippocampal Inflammation. Front Pharmacol. 2017囚8:886.

8 Poor ZNKM, Roodneshin F, Ahmadi DN, Gerami E, Riahi E. Early childhood exposure to short periods of sevoflurane is not associated with later, lasting cognitive deficits. Paediatr Anaesth. 2016冈26:1018-25.

9 Sun LS, Li G, Dimaggio C, Byrne M, Rauh V, Brooks-Gunn J, et al. Anesthesia and neurodevelopment in children: time for an answer. Anesthesiology. 2008®109:757-61.

10 Bo LJ, Yu PX, Zhang FZ, Dong ZM. Dexmedetomidine mitigates sevoflurane-induced cell cycle arrest in hippocampus. J Anesth. 2018®32:717-24.

11 Kim BS, Choi JH, Baek SH, Lee DH. Effects of Intraneural Injection of Dexmedetomidine in Combination With Ropivacaine in Rat Sciatic Nerve Block. Reg Anesth Pain Med. 2018®43:378-84.

12 Brummett CM, Norat MA, Palmisano JM, Lydic R. Perineural administration of dexmedetomidine in combination with bupivacaine enhances sensory and motor blockade in sciatic nerve block without inducing neurotoxicity in rat. Anesthesiology. 2008ه109:502-11.

13 Alam A, Suen KC, Hana Z, Sanders RD, Maze M, Ma D. Neuroprotection and neurotoxicity in the developing brain: an update on the effects of dexmedetomidine and xenon. Neurotoxicol Teratol. 2017区 60:102-16.

14 Wang L, Liu H, Zhang L, Wang G, Zhang M, Yu Y. Neuroprotection of Dexmedetomidine against Cerebral Ischemia-Reperfusion Injury in Rats: Involved in Inhibition of NF-KB and Inflammation Response. Biomol Ther (Seoul). 2017®25:383-9.

15 Yin D, Zhou S, Xu X, Gao W, Li F, Ma Y, et al. Dexmedetomidine attenuated early brain injury in rats with subarachnoid haemorrhage by suppressing the inflammatory response: The TLR4/NF-KB pathway and the NLRP3 inflammasome may be involved in the mechanism. Brain Res. 2018ه1698:1-10.

16 Qian XL, Zhang W, Liu MZ, Zhou YB, Zhang JM, Han L, et al. Dexmedetomidine improves early postoperative cognitive dysfunction in aged mice. Eur J Pharmacol. 20158746:206-12. 
17 Yi C, Fu Z, Luo X. Dexmedetomidine on autophagy of hippocampal neurons in aged rats under sevoflurane anesthesia. Exp Ther Med. 2018®16:837-41.

18 Umholtz M, Nader ND. Anesthetic Immunomodulation of the Neuroinflammation in Postoperative Cognitive Dysfunction. Immunol Invest. 2017ه46:805-15.

19 Cortese GP, Burger C. Neuroinflammatory challenges compromise neuronal function in the aging brain: Postoperative cognitive delirium and Alzheimer's disease. Behav Brain Res. 2017凶322:269-79.

20 Pol RA, van Leeuwen BL, Izaks GJ, Reijnen MM, Visser L, Tielliu IF, et al. C-reactive protein predicts postoperative delirium following vascular surgery. Ann Vasc Surg. 2014『28:1923-30.

21 Eger El. New inhaled anesthetics. Anesthesiology. 1994『80:906-22.

22 Esper T, Wehner M, Meinecke CD, Rueffert H. Blood/Gas partition coefficients for isoflurane, sevoflurane, and desflurane in a clinically relevant patient population. Anesth Analg. 2015ه120:45-50.

23 Lee YM, Song BC, Yeum KJ. Impact of Volatile Anesthetics on Oxidative Stress and Inflammation. Biomed Res Int. 2015囚2015:242709.

24 Zhang L, Zhang J, Yang L, Dong Y, Zhang Y, Xie Z. Isoflurane and sevoflurane increase interleukin-6 levels through the nuclear factor-kappa B pathway in neuroglioma cells. Br J Anaesth. 2013ه110 Suppl $1:$ i82-91.

25 Ko JK, Auyeung KK. Target-oriented mechanisms of novel herbal therapeutics in the chemotherapy of gastrointestinal cancer and inflammation. Curr Pharm Des. 2013®19:48-66.

26 Ma XM, Blenis J. Molecular mechanisms of mTOR-mediated translational control. Nat Rev Mol Cell Biol. 2009ه10:307-18.

27 Iadevaia V, Huo Y, Zhang Z, Foster LJ, Proud CG. Roles of the mammalian target of rapamycin, mTOR, in controlling ribosome biogenesis and protein synthesis. Biochem Soc Trans. 2012 40:168-72.

28 Albert V, Hall MN. mTOR signaling in cellular and organismal energetics. Curr Opin Cell Biol. 2015区 33:55-66.

29 Xu L, Brink M. mTOR, cardiomyocytes and inflammation in cardiac hypertrophy. Biochim Biophys Acta. 2016ه1863:1894-903.

30 Zhang Y, Nicholatos J, Dreier JR, Ricoult SJ, Widenmaier SB, Hotamisligil GS, et al. Coordinated regulation of protein synthesis and degradation by mTORC1. Nature. 2014囚513:440-3.

31 Harston RK, McKillop JC, Moschella PC, Van Laer A, Quinones LS, Baicu CF, et al. Rapamycin treatment augments both protein ubiquitination and Akt activation in pressure-overloaded rat myocardium. Am J Physiol Heart Circ Physiol. 2011®300:H1696-706. 
32 Tak PP, Firestein GS. NF-kappaB: a key role in inflammatory diseases. J Clin Invest. 2001ه107:7-11.

33 Yang J, Li S, Wang L, Du F, Zhou X, Song Q, et al. Ginsenoside Rg3 Attenuates LipopolysaccharideInduced Acute Lung Injury via MerTK-Dependent Activation of the PI3K/AKT/mTOR Pathway. Front Pharmacol. 2018®9:850.

34 Chen Z, Qiu PY, Ma CG. Dexmedetomidine preconditioning protects against retinal ischemia/reperfusion injury and inhibits inflammation response via toll-like receptor 4 (TLR4) pathway. Biomed Pharmacother. 2017ه93:1018-24.

35 Shen M, Wang S, Wen X, Han XR, Wang YJ, Zhou XM, et al. Dexmedetomidine exerts neuroprotective effect via the activation of the PI3K/Akt/mTOR signaling pathway in rats with traumatic brain injury. Biomed Pharmacother. 2017ه95:885-93.

\section{Figures}




\section{cortex}
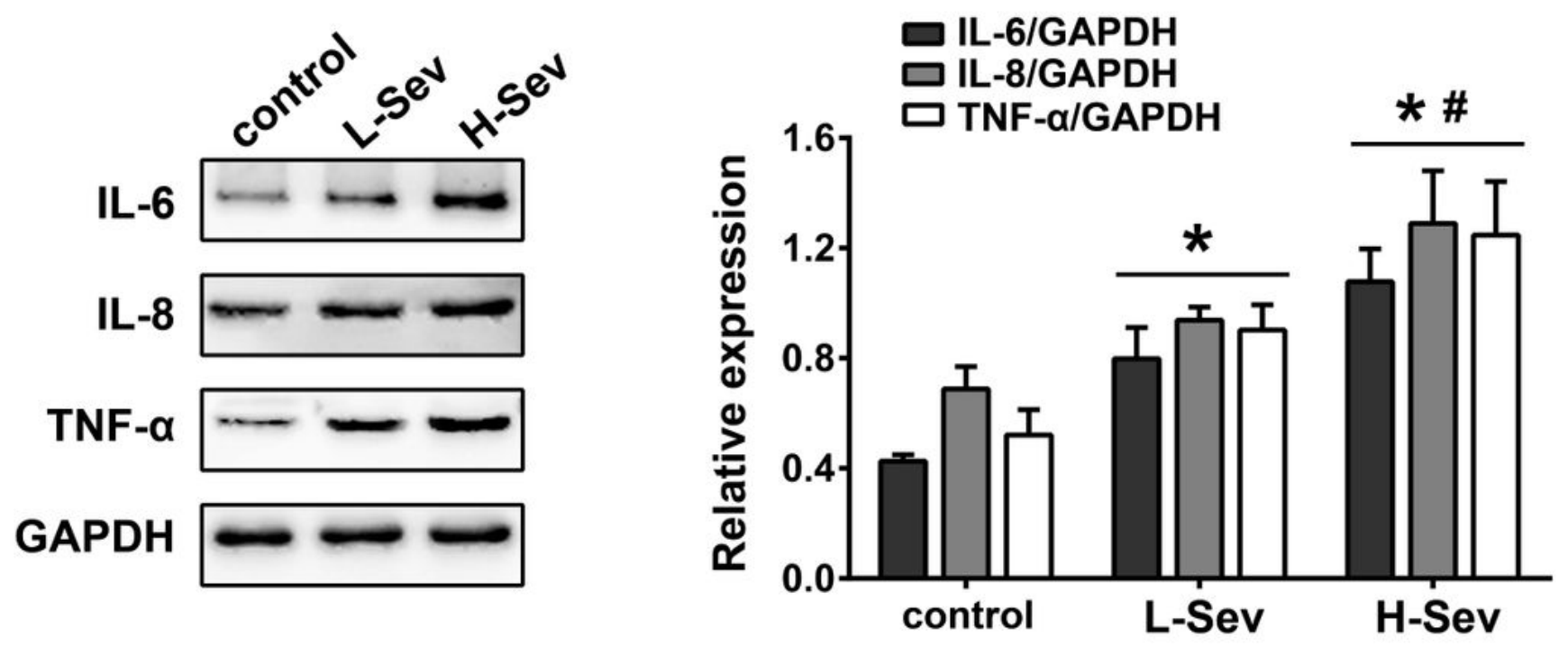

hippocampus
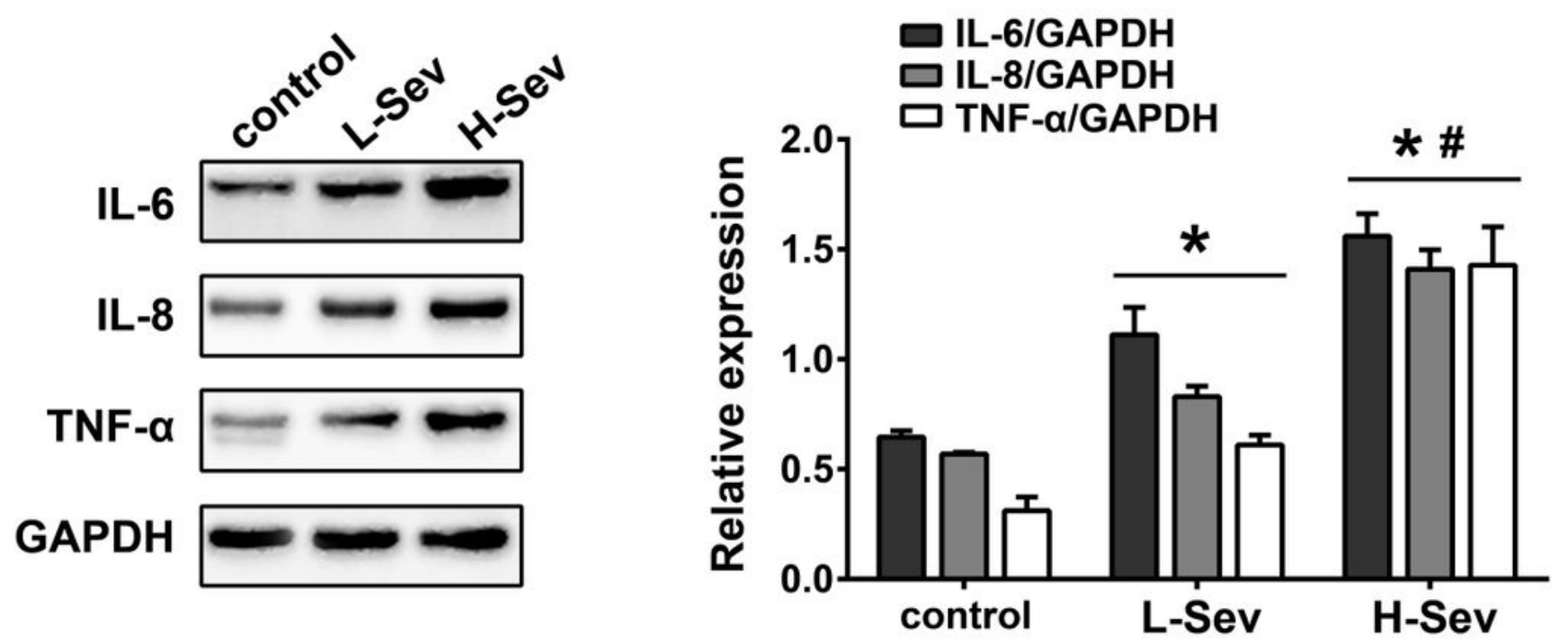

Figure 1

Sevoflurane treatment increases inflammatory markers in the hippocampus. Expression levels of IL-6, IL-8 and TNF-a in the cortex and hippocampus of rats in the control, L-Sev, and H-Sev groups were measured by 
cortex

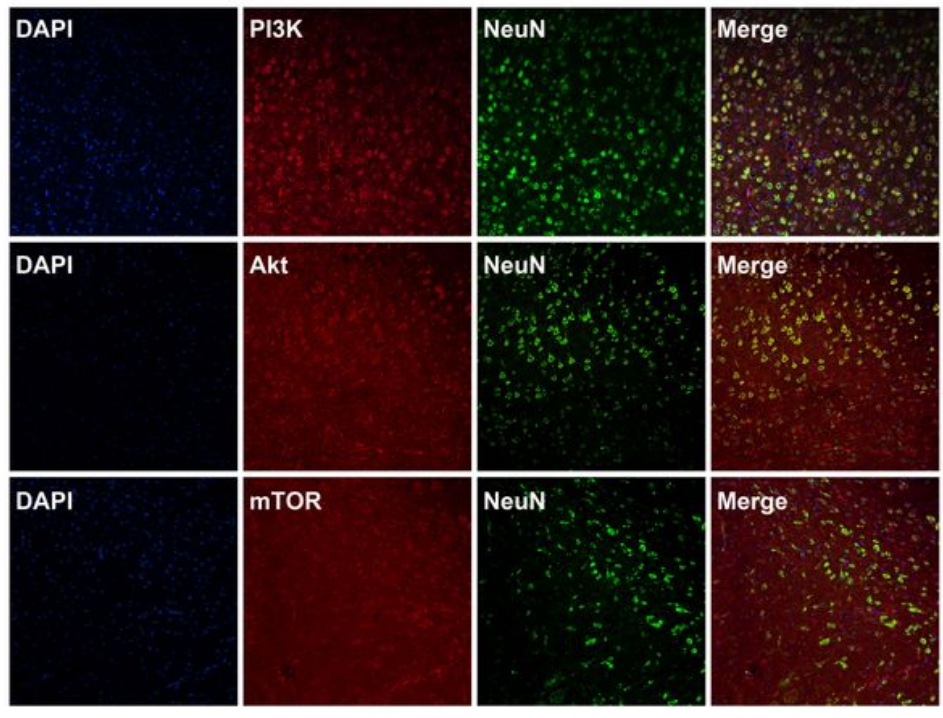

hippocampus

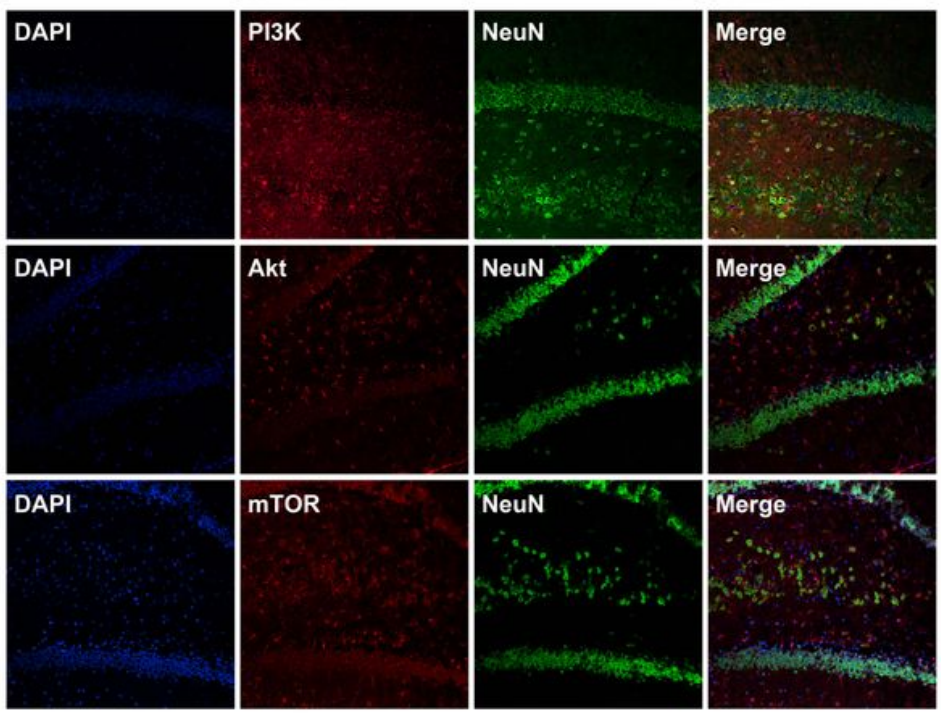

Figure 2

Immunofluorescence staining for PI3K/Akt/mTOR pathway components. PI3K, Akt, and mTOR are widely expressed in neurons and other non-neuronal cells in the cortex and hippocampus (magnification 200x). 
cortex
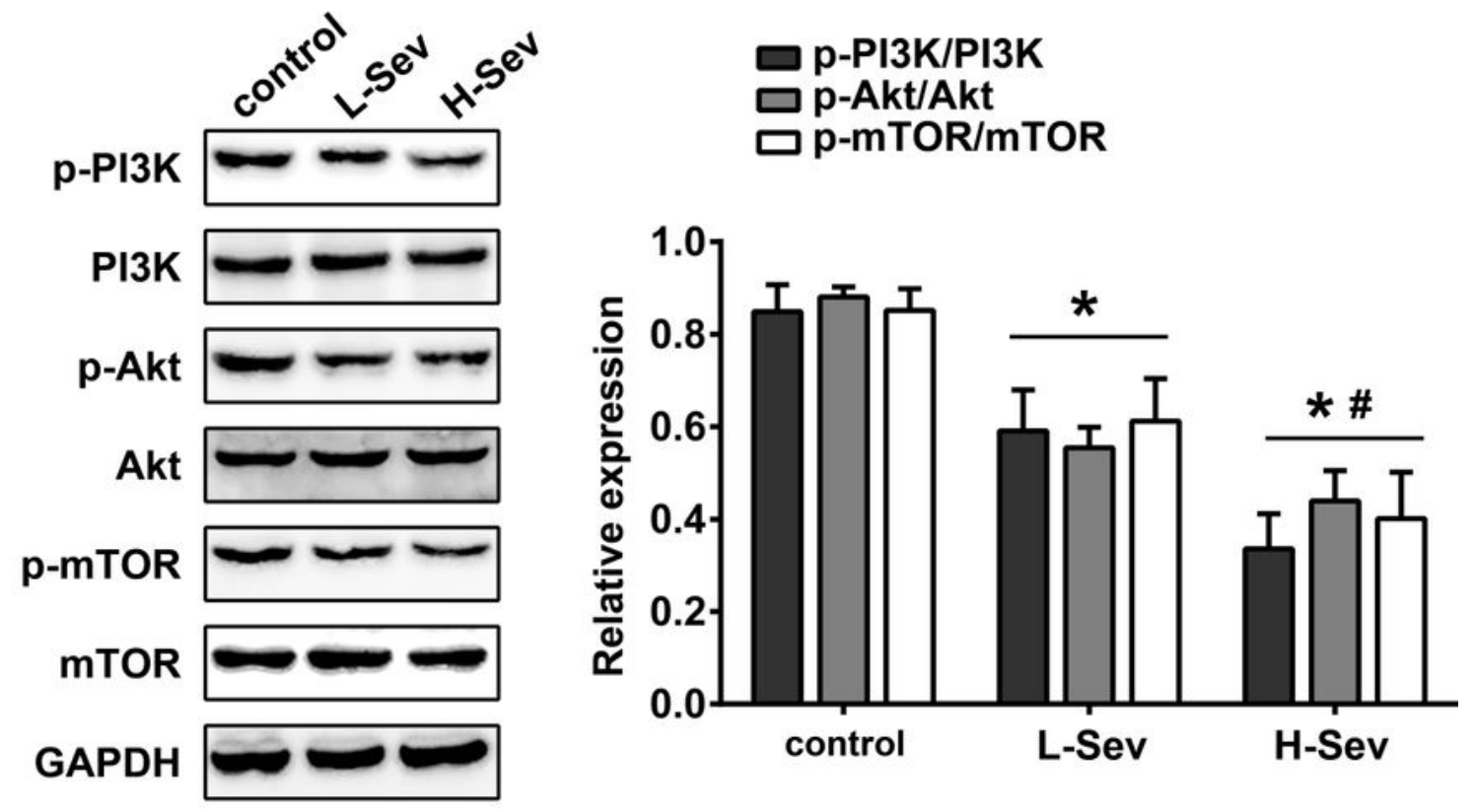

hippocampus
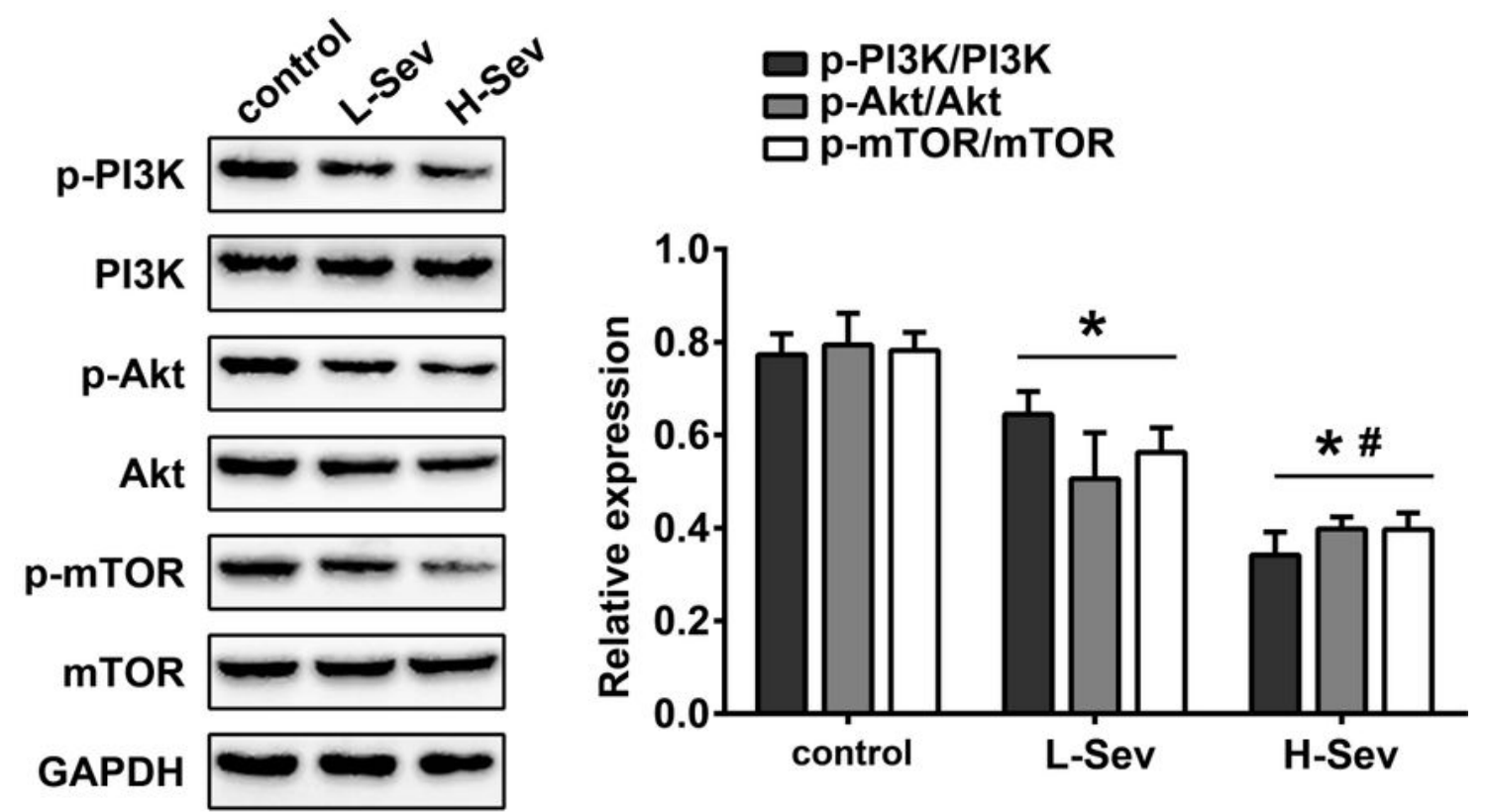

Figure 3

Effect of dexmedetomidine on the PI3K/Akt/mTOR pathway Images and quantification of western blot measurements of PI3K, p-PI3K, Akt, p-Akt, mTOR and p-mTOR in the cortex and hippocampus of rats in the vehicle and $D E X$ groups (mean $\pm S D, n=5$ per group, $t$ test). ${ }^{*}<0.05$ vs vehicle group. 


\section{cortex}
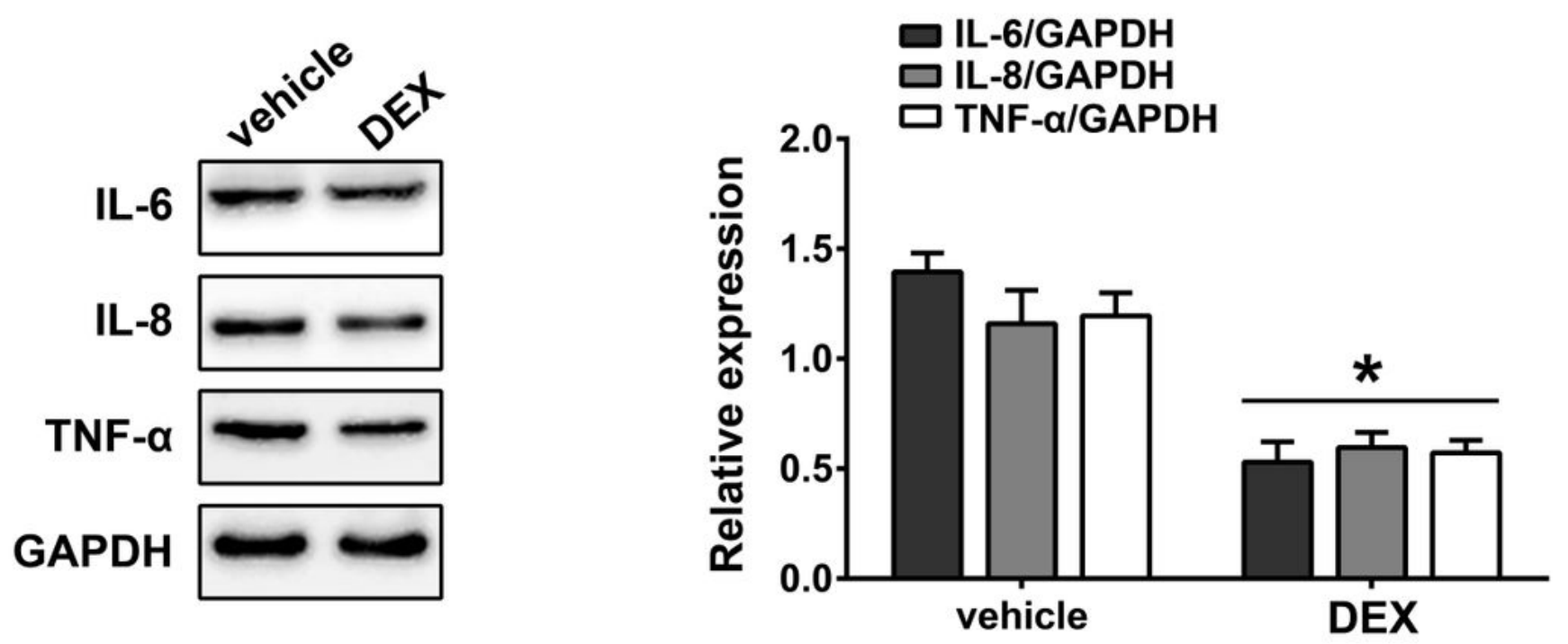

\section{hippocampus}
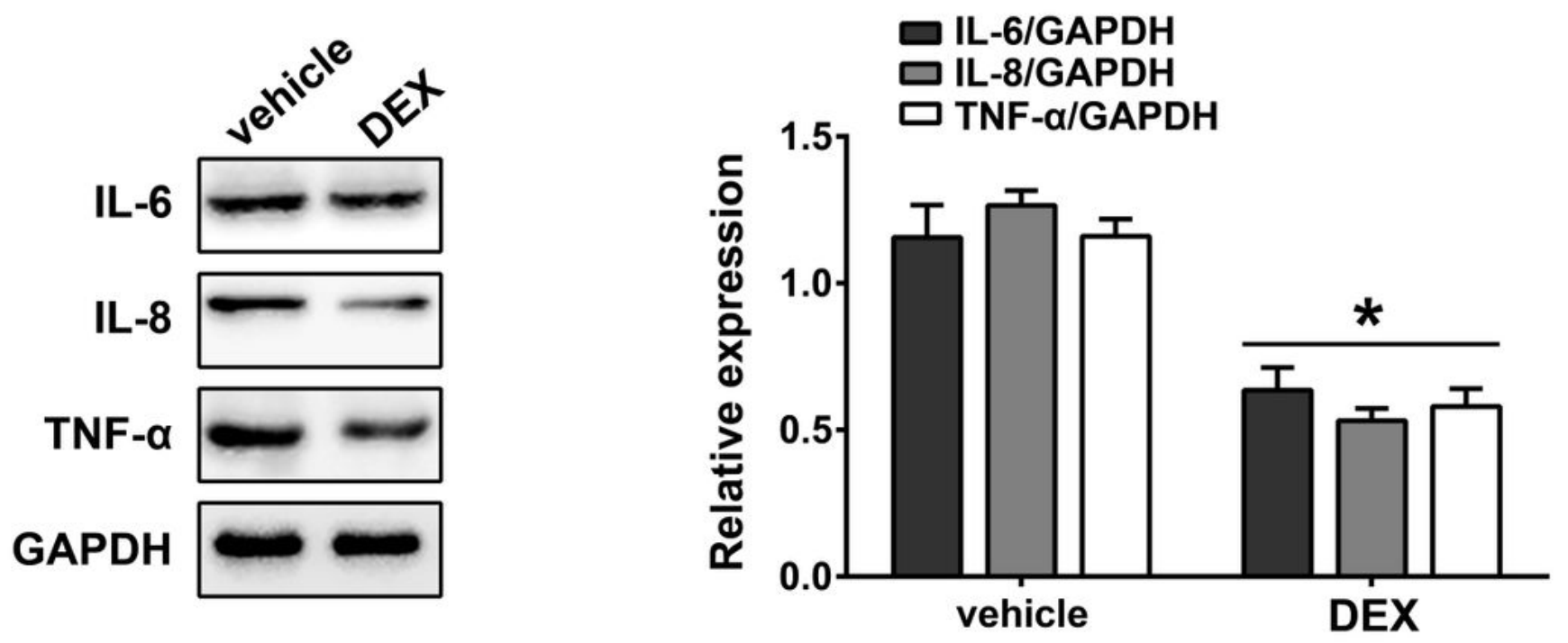

Figure 4

Effect of sevoflurane on PI3K/Akt/mTOR pathway activity. Cortical and hippocampal levels of PI3K, pPI3K, Akt, p-Akt, mTOR and p-mTOR of rats in the control, L-Sev and H-Sev groups were measured by western blotting (mean $\pm S D, n=5$ per group, one-way ANOVA). ${ }^{\star} p<0.05$ vs control group, $\# p<0.05$ vs L-Sev group. 
cortex
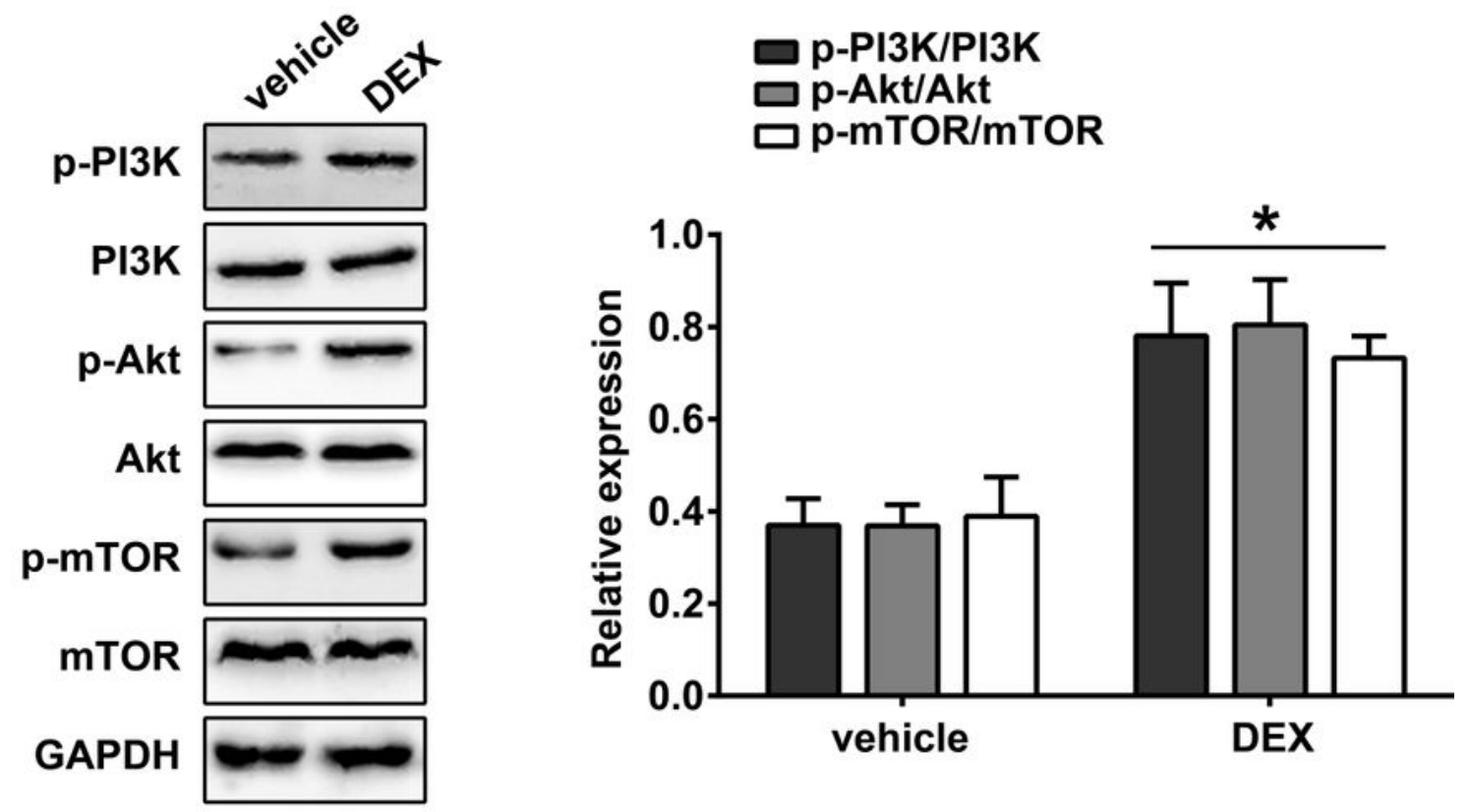

hippocampus
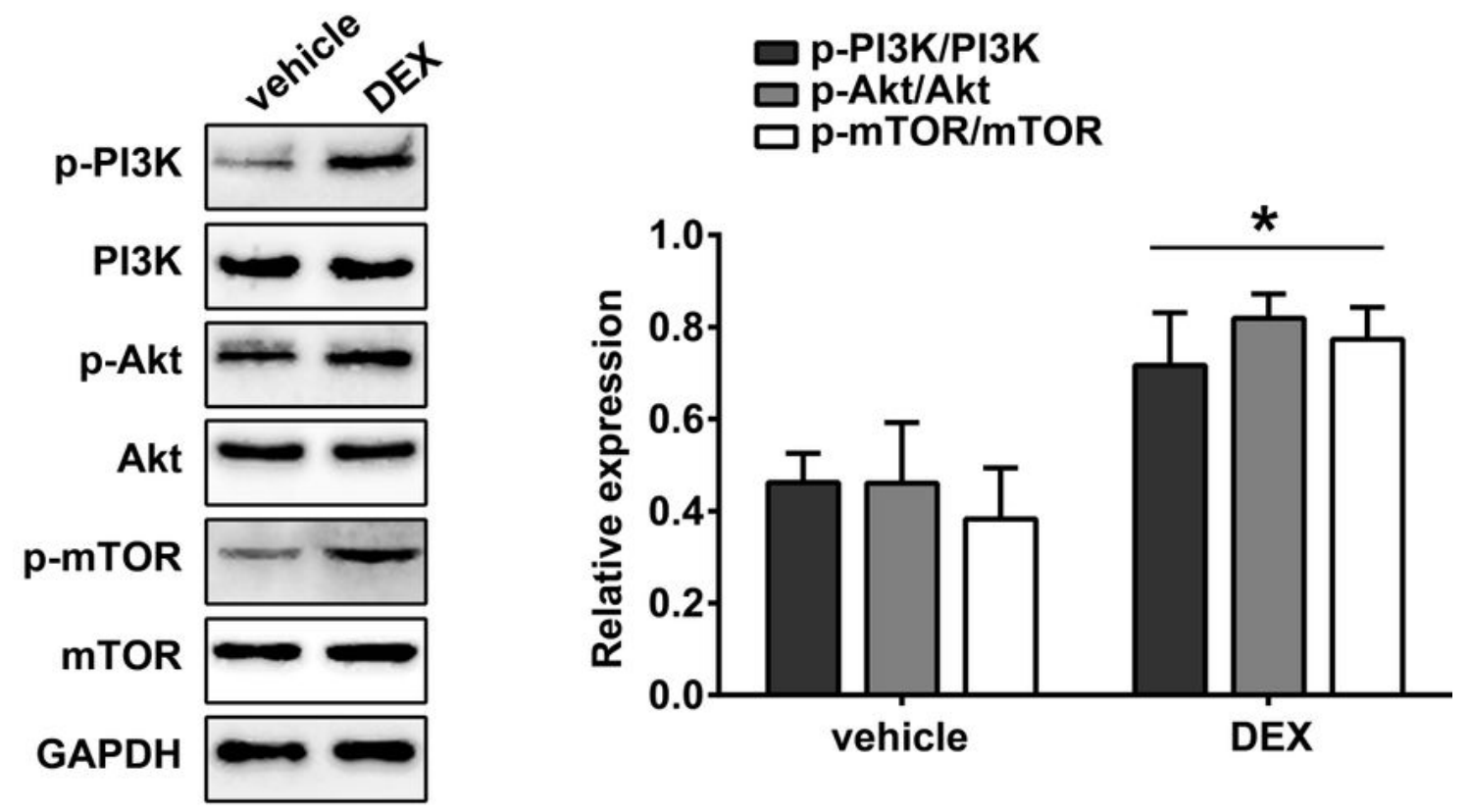

Figure 5

Effect of dexmedetomidine on sevoflurane anesthesia-induced neuroinflammation Western blot measurements of IL-6, IL-8, and TNF- a from cortical and hippocampal samples of DEX and vehicle treated rats (mean $\pm S D, n=5$ per group, $t$ test). ${ }^{*} p<0.05$ vs vehicle group. 
This is a list of supplementary files associated with this preprint. Click to download.

- supplement1.pdf 